\title{
Research of vibration asymmetry of fluidic oscillator with vortex chambers
}

\author{
Kazimierz Peszynski ${ }^{1, a}$, Emil Smyk ${ }^{1}$, Sylwester Wawrzyniak ${ }^{1}$, and Daniel Perczynski ${ }^{1}$ \\ ${ }^{1}$ UTP University of Science and Technology in Bydgoszcz, Faculty of Mechanical Engineering, Control Group, Poland
}

\begin{abstract}
The paper presents an analysis of the test results of a new type of fluidic oscillator. The oscillator design is based on the internal feedback in the form of two vortex chambers. The results were transformed into a dimensionless form. Then, the effect of deliberately introduced asymmetry into one of two input channels of the feedback chambers on the device oscillation asymmetry was examined.
\end{abstract}

\section{Introduction}

In the fluidic oscillators, periodic oscillation of fluid passing through a device can be induced without moving elements. The effect is obtained by suitable shaping of channels and chambers where fluid (mostly air) goes through. The fluidic oscillator is characterised by the input feedback.

The typical oscillator (Fig. 1) consists of the following elements: the supply channel, nozzle, separator chamber with specified inclination of walls, splitter etc. The feedback circuit is usually a channel which connects one of the output channels (in case of oscillators with two outputs) or working chamber (in case of oscillators with one output) with the interaction region.

Oscillators with the feedback have already been described in the 1980s (Belsterling, 1971, Davies, 1970, Tippetts et al., 1973) - Fig. 1. The jet from the nozzle attracts to one of two walls of the operating cavity as a result of the Coanda effect and flows in the direction of the output channel. Before leaving the cavity, the stream is divided into two parts on the splitter of suitable shaped separator. Most of the fluid flows out through the output channel, the rest of it flows into the feedback channel. After passing through the channel, the stream gets the required speed and affects the adjacent input stream and moves it towards the second wall. The process of the stream separation repeats in the second part of chamber, which results in relocation of the stream towards the other wall. The phenomenon leads to the pulsation of pressure in both output channels (Fig.1a) or one output channel (Fig. 1b). It is common for this type of oscillator to have two output channels and the stream flows out alternately from one or the other channel. a)

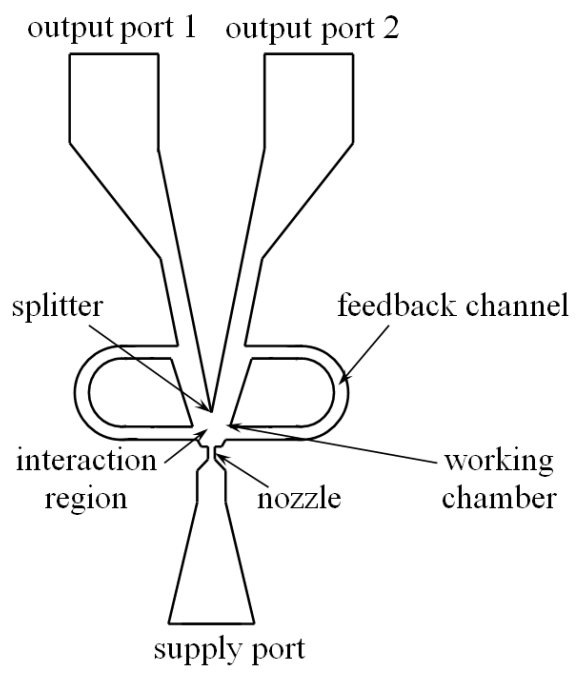

b)

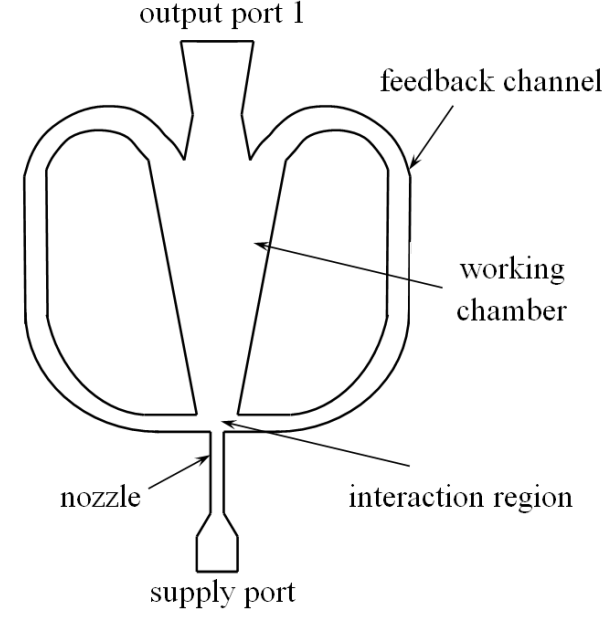

Figure 1. Characteristic parts of the classic oscillator with feedback: a) with two outputs, b) with one output

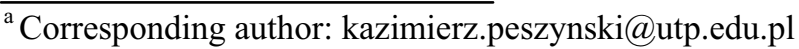


The following characteristic numbers play the key role in the design, analysis and mathematical modelling of the fluidic elements: Strouhal number $(\mathrm{Sr})$, Reynolds number $(R e)$, Euler number $(E u)$, and the dimensionless parameter of the feedback circuit $\left(L^{\prime}\right)$, which can be calculated from the equations:

$$
\begin{gathered}
S r=\frac{f h}{u} \\
R e=\frac{u h}{v} \\
E u=\frac{2 \Delta p}{\rho u^{2}} \\
L^{\prime}=\frac{4 l^{\prime} n}{\pi d^{\prime 2}}
\end{gathered}
$$

where $h$ is the characteristic dimension of the oscillator, $f$ - the frequency of pressure oscillation, $u$ - the speed of fluid at the entry, $v$ - the kinematic viscosity, $\Delta p-$ the pressure drop, and $\rho$ - the fluid density. Furthermore, $l^{\prime}=l / d_{\mathrm{n}}$ and $d^{\prime}=d / d_{\mathrm{n}}$, where $l$ and $d$ are the length and diameter of the feedback channel. $d_{\mathrm{n}}$ and $n$ are the width and shape factor of the output channel of the circuit.

\section{Research subject and test stand}

The subject of the study was a new type of fluidic oscillator without a classic feedback circuit (Figure 2).

a)

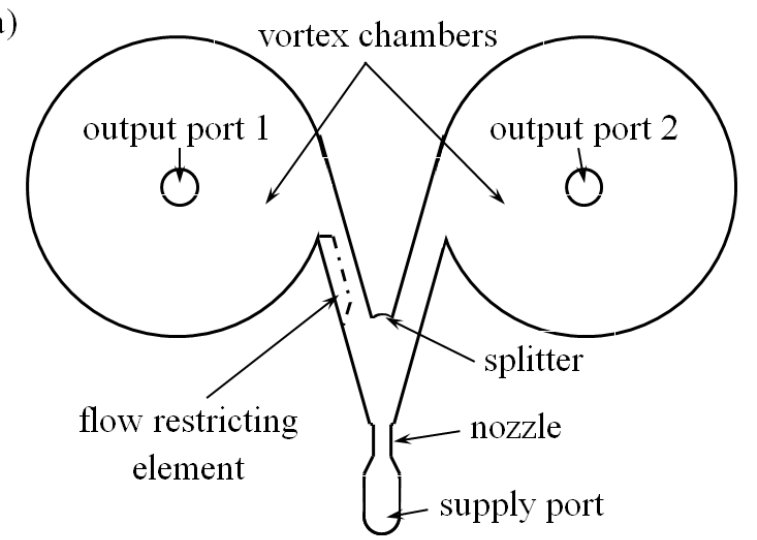

b)

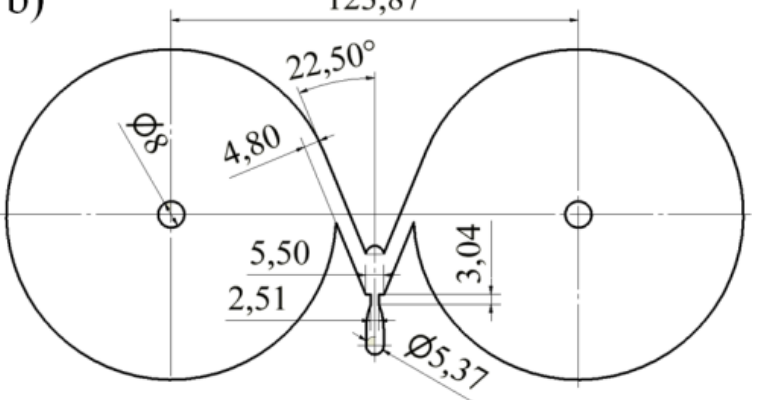

Figure 2. Tested oscillator: a) elements of the oscillator, b) characteristic dimensions

The preliminary test results of the oscillator, designed in the Institute of Thermomechanics of Academy of Science of Czech Republic by Professor Tesař, has been presented in the paper by Tesař and Smyk (2015b). The feedback channels were replaced with the vortex chambers, which are located at the end of each output channel of interaction region. Increasing pressure in the vortex chambers plays the role of the feedback. As a result of Coanda effect, the stream from the oscillator attracts to one of the walls and goes to the vortex chamber. It starts to move in rotational motion, which causes the accumulation of fluid mass and increase of pressure. As a result of increased resistance, the stream is moved to the second vortex chamber with a lower pressure. During the filling of the second chamber, some of accumulated fluid in the first chamber leaves it through the output port located in the middle. The phenomenon repeats in the second chamber, resulting in an oscillation in both output ports of the oscillator.

The shape of tested oscillator is presented in Figure $2 \mathrm{~b}$. The oscillator chamber was made of PMMA plate, $7.8 \mathrm{~mm}$ thick. The fluid stream flows into the device through the supply port with a connection tube, $5.37 \mathrm{~mm}$ diameter. At the end of the input channel, there is a nozzle of rectangular cross-section, $2.51 \mathrm{~mm}$ width. After flowing through the nozzle, the gas attracts to one of the working chamber walls and flows through the channel, $4.80 \mathrm{~mm}$ width, and gets into one the vortex chambers.

The air was delivered from a tank with a capacity of $28 \cdot 10^{-3} \mathrm{~m}^{3}$ DURR-DENTAL reciprocating compressor. The tank suppressed pressure pulsation of the supply air. The measurement and setting of the gas flow rate was made by VEB MLW PRUFGERATER-WERK float (glass) rotameter with an operating distance of $0.2 \div 2$ and measurement accuracy of $0.010 \mathrm{~m}^{3} \cdot \mathrm{h}^{-1}$. The speed of gas at the oscillator outlet was measured by HWA (HotWire Anemometer) thermo-anemometer with the use of Constant Temperature Anemometer (CTA) single-sensor wire probe, No. 55P16, DANTEC company. The tests were taken for the following parameters of the gas: absolute number $T=295 \mathrm{~K}$, ambient pressure $p=102500 \mathrm{~Pa}$, and relative humidity $\varphi=28 \%$.

\section{Research results}

Before the main tests, there were carried out preliminary tests of the oscillator without elements which limit the flow of one of the channels behind the separation chamber. The tests were used to verify actions of the oscillator and to test the methods of results development.

The next step was to place in the left channel behind the separation funnel, the elements which limit $A_{k}$ crosssection.

\subsection{Preliminary studies}

Figure 3 shows the process of outflow gas speed at the oscillator output port for selected rate of the supply stream velocity. The measured flow is unsettled. However, the tendency to form the output sinusoidal stream can be noted. The Fast Fourier Transform (FFT) was used in order to determine the approximating frequency of the oscillator. 4096 samples were treated for 
8 flow rates in the range of $(166.7 \div 555.6) \cdot 10^{-6} \mathrm{~m}^{3} / \mathrm{s}$, graduated at $66.7 \cdot 10^{-6} \mathrm{~m}^{3} / \mathrm{s}$. The selected time and sampling frequency allowed determining the frequency of approximating signal with accuracy of $f_{\min }=0.5 \mathrm{~Hz}$. For the case presented in Figure 3, the period was obtained as $T=0.182 \mathrm{~s}$ and the amplitude as $A=2.63 \mathrm{~m} / \mathrm{s}$.

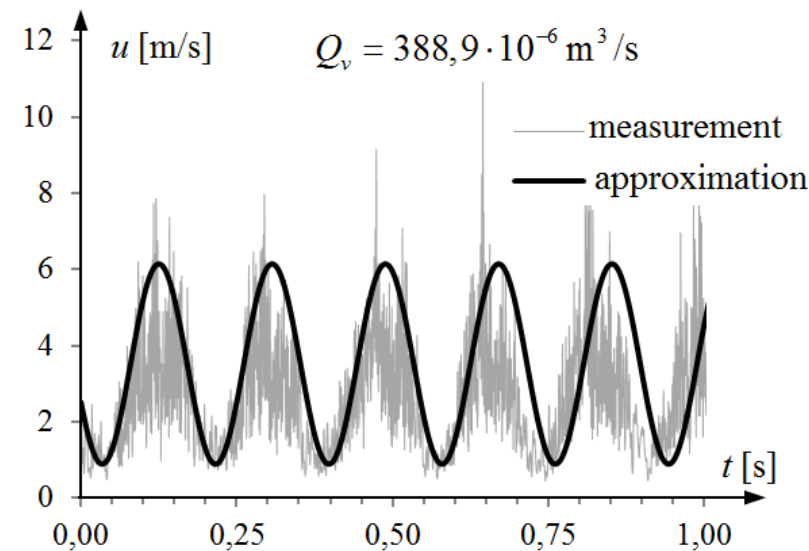

Figure 3. The course of speed at the oscillator outlet with sinusoidal process

For a given range of the flow rates of the supply stream, characteristic was drawn and presented in Figure 4.

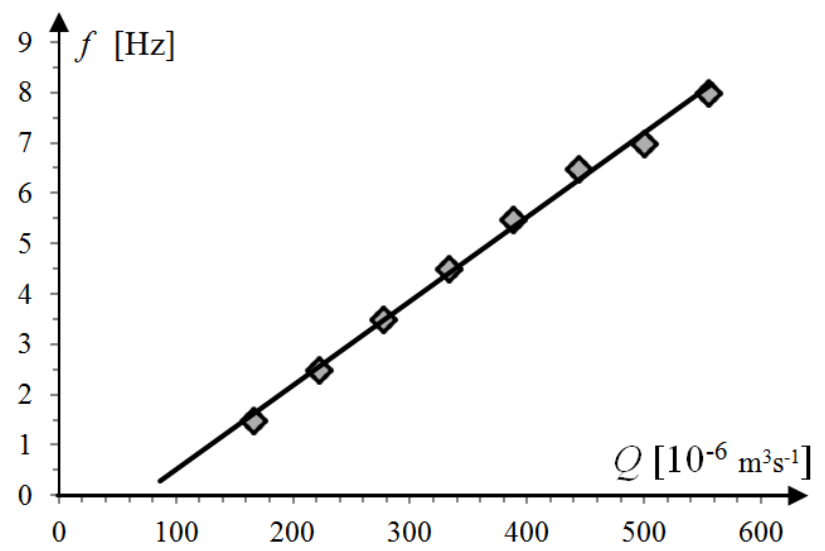

Figure 4. Approximated and real frequencies versus flow rate function

The obtained dependence is assumed to be linear, but it does not go through the beginning of the coordinate system, so:

$$
f=a Q_{V}+b=a A_{\mathrm{k}} u_{\mathrm{sr}}+b
$$

After linear approximation, the slope of the straight line was determined as $a=16714$ and the intercept of a line $b=-1.1607$.

In order to generalise the results of research, the results were presented in the form of dimensionless numbers $S r=f(R e)$. To calculate Strouhal number $(S r)$ and Reynolds number $(R e)$, the characteristic dimension of the channel width was used with the initial value of $h=4.8 \mathrm{~mm}$, as well as average speed of the stream at the channel input of the oscillator interaction region, which was determined from the equation $Q_{V}=A_{k} u_{s r}$.

The kinematic viscosity was calculated from the equation

$$
v=\frac{\mu}{\rho}
$$

where the dynamic viscosity $\mu$ was given from the equation

$$
\mu=\frac{1.458 \cdot 10^{-6} \cdot T_{L}^{0,5}}{1+\frac{110.4}{T_{P}}}
$$

It was assumed that the temperature of the working medium $T_{P}$ is approximately equal to the temperature in the laboratory.

The specific mass $\rho$ was determined from the equation

$$
\rho=\frac{p_{b a r}}{r \cdot T_{P}}
$$

where the individual gas constant for air was

$$
r=\frac{0.426 \cdot \psi+287}{1+0.001 \cdot \psi}
$$

the specific humidity

$$
\psi=\frac{622 \cdot E}{p_{b a r}-0.378 \cdot E}
$$

the saturated vapour pressure was calculated from Mangus equation

$$
E=610 \cdot 10^{\frac{7.5 \cdot\left(T_{L}-273.15\right)}{T_{L}-38.15}}
$$

When multiplied the equation (5) by the factor $h / u_{s r}$, we obtain:

$$
\frac{f h}{u_{\mathrm{sr}}}=\frac{\left(a A_{k} u_{\mathrm{sr}}+b\right) h}{u_{\mathrm{sr}}}=a A_{k} h+b h \frac{1}{u_{\mathrm{sr}}}
$$

Based on the equation (2), it is possible to determine the average air speed $u_{\mathrm{sr}}$ as $u_{\mathrm{sr}}=\frac{v R e}{h}$. After substituting the equation (12) on the left side with the equation (1) and $u_{\mathrm{sr}}=\frac{v R e}{h}$ on the right side, we obtain

$$
S r=a A_{k} h+\frac{b h^{2}}{v \operatorname{Re}}
$$

Substituting the given numerical values of $A_{k}=2,827 \cdot 10^{-5} \mathrm{~m}^{2}$ and $v=1,518 \cdot 10^{-5} \mathrm{~m}^{2} \mathrm{~s}^{-1}$ into equation (13), we obtain

$$
S r=2,2684 \cdot 10^{-3}-1,76148 \frac{1}{R e}
$$




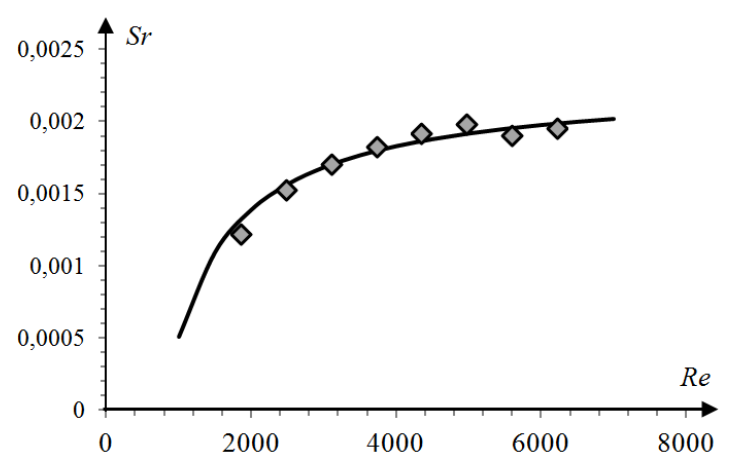

Figure 5. Characteristics of the oscillator with the vortex chamber - dependence between Strouhal number and Reynolds number

The range of possible to achieve frequencies is limited in practical solution. The increase of the flow rate is impossible, because hydraulic resistance increases rapidly, which causes significant pressure drop. The phenomenon is unfavourable in series connections of the stream elements.

\subsection{Asymmetric oscillations}

The research of the oscillator asymmetry was carried out because of three reasons:

- determining the influence of the asymmetry on the output frequency of the oscillator,

- determining the possible impact of the errors on the actions of the oscillator,

- determining the dependence between the cross-section asymmetry of the feedback input channels and the output frequency.

The oscillator asymmetry was obtained by reducing the cross-section of one of the input channels to the vortex chambers (Figure 2a by permanently placing an element which reduces the cross-section in the channel. The minimum geometric dimensions of the channel in the plane of reduced cross-section, for different cases, are shown in Table 1.

Table 1. Dimension of the channel for different settings of experiment

\begin{tabular}{|c|c|}
\hline Percentage of cross-section & Channel dimensions \\
\hline $100 \%$ of cross-section & $4.80 \times 7.80 \mathrm{~mm}$ \\
\hline $80 \%$ of cross-section & $3.84 \times 7.80 \mathrm{~mm}$ \\
\hline $60 \%$ of cross-section & $2.88 \times 7.80 \mathrm{~mm}$ \\
\hline $40 \%$ of cross-section & $1.92 \times 7.80 \mathrm{~mm}$ \\
\hline $20 \%$ of cross-section & $0.96 \times 7.80 \mathrm{~mm}$ \\
\hline $0 \%$ of cross-section & Channel fully blocked \\
\hline
\end{tabular}

Figure 6 presents the characteristic of the oscillator in the output of the vortex chamber, supplied by the channel with unchanged cross-section. The cross-section of the other channel was changed in accordance to the Table 1.

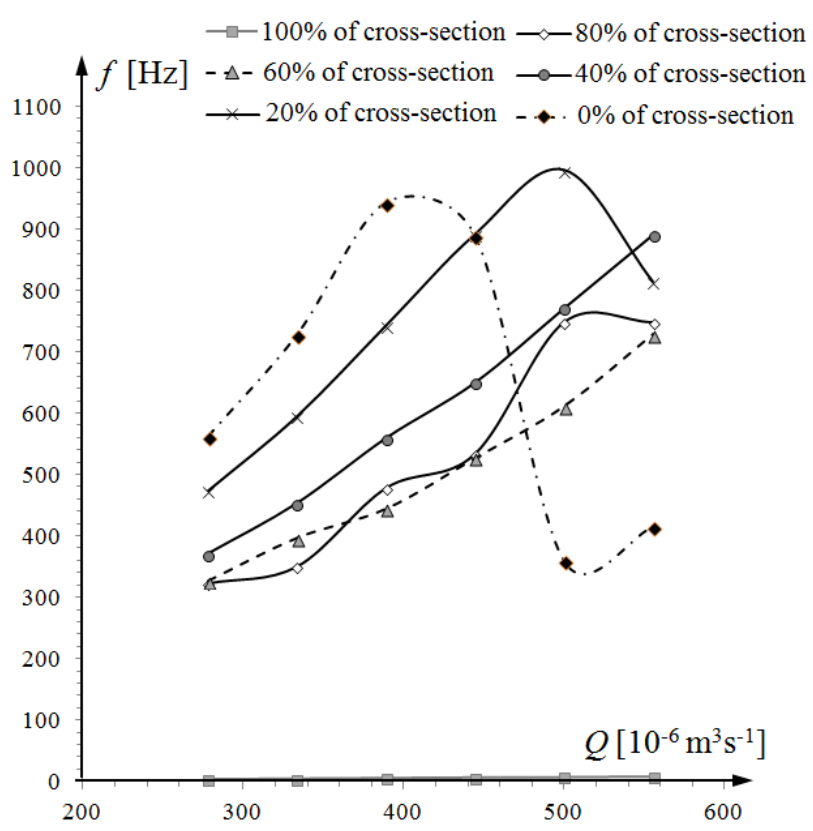

Figure 6. Oscillator asymmetry for the output channel with unchanged input cross-section

On the output of the other chamber (Figure 2a), the oscillation frequency significantly increases with the implementation of the asymmetry. The oscillation frequency dependence from the power flow rate $f_{2}=f\left(Q_{V}, x\right)$ is approximately linear, at least for several ranges of the volumetric flow rate. This allows the possibility of precise control of the output signal. The oscillation frequencies $f$ of the output signals for two channels (one with the reduced cross-section and the other one with unchanged cross-section) have different values, but they are different in both cases, which is related directly to the difference of the flow rate in each channel. It should be noted that reducing the crosssection area of the channel is equivalent to its throttling, which means reduction of the flow rate.

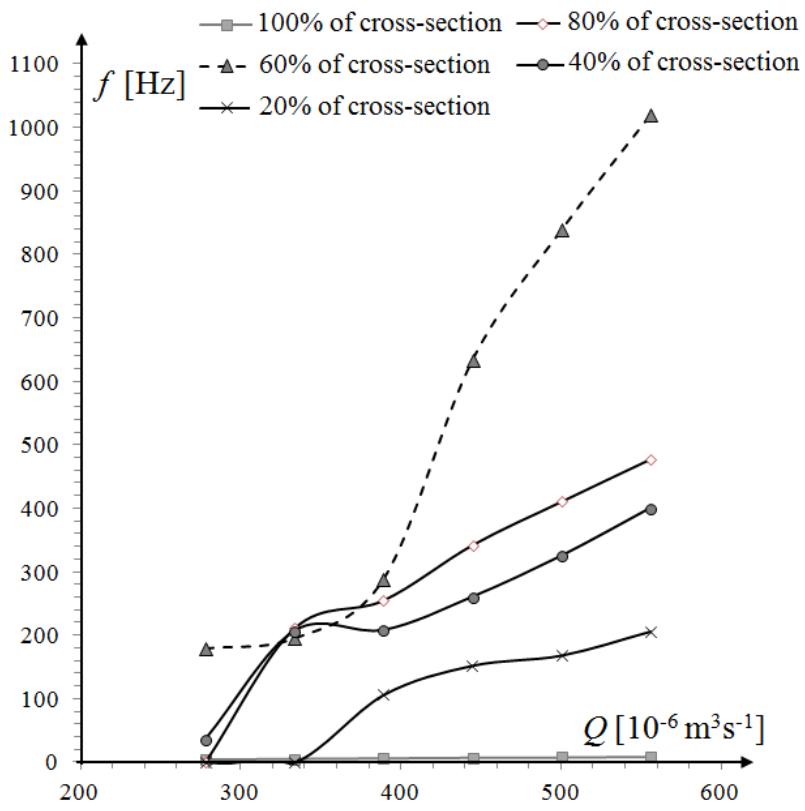

Figure 7. Oscillator asymmetry; the output channel with reduced input cross-sections 


\subsection{Frequency spectrum analysis}

The frequency spectrum analysis, obtained by the fast Fourier transform (FFT), allows analysing the quality of received signals. Figure 8 presents the spectrum obtained for the output signal in case of the oscillator with unchanged input cross-section (Figure 8a), as well as the spectrum obtained on the output of the channel with an element which limits the cross-section of the other channel of $40 \%$ of initial cross-section (Figure $8 b$ ).
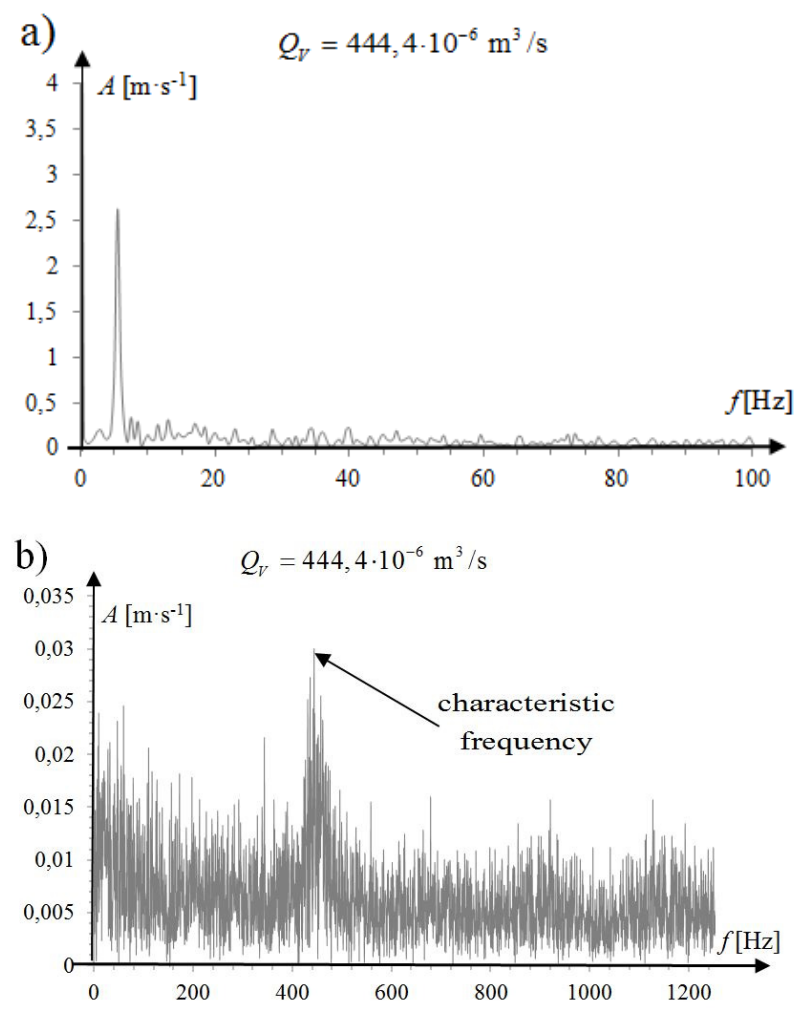

Figure 8. Analysis of the frequency spectrum, input value, oscillator, a) for the channel without damping mass, b) for the channel without intalled mass in the cross-section of the second channel of $40 \%$ of initial cross-section

The frequency spectrum in the first case shows the oscillation frequency equal $f=5,5 \mathrm{~Hz}$, without significant noise for other frequencies. In the case of installing mass, which limits the cross-section, in the oscillator, the frequency analysis shows a considerable share of the noise in the output signal. This phenomenon is unwanted. Moreover, the characteristic frequency amplitude is slight (approx. $A=0,04 \mathrm{~m} / \mathrm{s}$ ). In these cases, the signal should be filtered and strengthened, which includes other operations.

\section{Conclusions}

The results confirm the possibility of changing the characteristics of the proposed oscillator by changing the cross-sectional area of one of the channels. The operation helped to increase the oscillation frequency of the oscillator output signal, without increasing the volumetric flow rate. Despite the increase in the noise level, the characteristic oscillation frequency can be found for selected parameters which limit the cross-section of one of two input channels. An important result of the study is the oscillation frequency of the output significantly increased.

\section{Acknowledgements}

Second author obtained support from Ministry of Science and Higher Educating of Poland in form of "Diamond Grant" 0201/DIA/2015/44 for young researchers.

There was also institutional support BS 16/2013 for all authors.

\section{References}

1. C.A. Belsterling, Fluidic Systems Design. John Wiley \& Sons, (1971)

2. R.C. Davies, Functional characteristics of fluid elements, Fluidics Quarterly 2.2, 1-43. (1970)

3. E.W. Simôes, R. Furlan, R.E.B. Leminski, M.R. Gongora-Rubio, M.T. Pereira, N.I. Morimoto, J.J.S. Avilés, Microfluidic oscillator for gas flow control and measurement. Flow Measurement and Instrumentation, 16(1), 7-12, (2005)

4. V. Tesař, C.H. Hung, W. B. Zimmerman, No-movingpart hybrid-synthetic jet actuator. Sensors and Actuators A: Physical, 125(2), 159-169 (2006)

5. V. Tesař, Configurations of fluidic actuators for generating hybrid-synthetic jets. Sensors and Actuators A: Physical, 138(2), 394-403 (2007)

6. V. Tesař, E. Smyk, Fluidic low-frequency oscillator with vortex spin-up time delay. Chemical Engineering and Processing: Process Intensification, 90, 6-15. (2015)

7. V. Tesař, E. Smyk, Infrasonic fluidic oscillator for use in anti-terrorist warfare. Safety and Security Engineering VI, 151, 179, (2015)

8. J.R. Tippetts, H.K. Ng, and J.K. Royle, An oscillating bistable fluid amplifier for use as a flowmeter, Fluidics Quarterly 5.1, 28-42, (1973)

9. J.T. Yang, C.K. Chen, K.J. W.Z. Tsai Lin,. H.J. Sheen, A novel fluidic oscillator incorporating stepshaped attachment walls. Sensors and Actuators A: Physical, 135(2), 476-483, (2007) 